\title{
Study on Needles and Cracks of Tin-doped Indium Oxide Tablets for Electron Beam Evaporation Process
}

\author{
Jiwen $\mathrm{Xu}^{a, b *}$, Zupei Yang ${ }^{b}$, Xiaowen Zhang ${ }^{a}$, Ling Yang ${ }^{c}$, Huarui X $u^{b}$, Hua Wang ${ }^{a}$ \\ ${ }^{a}$ Guangxi Key Laboratory of Information Materials, Guilin University of Electronic Technology, \\ Guilin 541004, P.R. China \\ ${ }^{b}$ School of Material Science and Engineering, Shaanxi Normal University, Xi'an 710062, P.R. China \\ ${ }^{c}$ Guangxi Experiment Center of Information Science, Guilin University of Electronic Technology, \\ Guilin 541004, P.R. China
}

Received: July 20, 2014; Revised: June 12, 2015

\begin{abstract}
Tin-doped indium oxide (ITO) tablets were used to deposit ITO films on p-GaN layer of lightemitting diodes. Needles and cracks in ITO tablets generated during electron beam evaporation process were deeply investigated. The formation of needles is predominantly resulted from the scanning trace, which is controlled by $x$ and $y$ axes scanning singles. The needles can be eliminated by controlling electron beam scanning trace. The loose microstructure with uniform grains and pores in the ITO tablets results in weak bonding strength, which leads to cracks under the thermal shock of high energy electron beam. A three-dimensional reticulated skeleton structure with strong bonding strength can restrain these cracks.
\end{abstract}

Keywords: needle, crack, ITO, electron beam evaporation

\section{Introduction}

Tin-doped indium oxide (ITO) films with high conductivity and transparency have been extensively served as transparent and conductive electrodes in optoelectronic devices ${ }^{1}$. There are some techniques to deposit the ITO films, such as magnetron sputtering, pulsed laser deposition, electron beam (EB) evaporation, chemical vapor deposition, sol-gel, spray pyrolysis ${ }^{2-7}$. Among these different methods, the EB evaporation technique is one of the most frequently used methods for producing ITO films having better electrical and optical properties, which is easy to be applied to commercial production $^{8}$. Some investigative works have reported the relationship between the EB evaporation process and the properties of the ITO films ${ }^{4,8-12}$, such as oxygen, postannealing, substrate temperature, ingredient ratio. One of the most important applications of the ITO films deposited by EB evaporation is served as the p-type electrodes of GaN-based light emitting diodes (LEDs). The utilization of the ITO films substituting for metal films can enhance the output of light from GaN-based LEDs ${ }^{13,14}$, which is due to its low direct current resistivity and high transmittance in the visible light range.

To the best of our knowledge, the high density ITO targets used for magnetron sputtering were served as the raw material of EB evaporation process. However, the ITO tablets designed for the source of EB evaporation were rarely investigated. In general, the low density of ITO tablets with about $62 \%$ relative density were designed to resist thermal shock of high energy electron beam. The surface needles or the internal cracks of the low density ITO tablets may be formed during EB evaporation process. Obviously, the

*e-mail: csuxjw@126.com formation of these needles or cracks are likely to change the scattering angel of the oxide vapor during evaporation process. These slight fluctuations would deteriorate the structural, electrical and optical properties of the deposited ITO films, such as resistivity, transmittance and uniformity. These deteriorated ITO films used for GaN-based LEDs would influence its electrical and optical properties.

In this work, the formation mechanism of the needles and cracks in the low density ITO tablets were investigated, and the corresponding solutions were proposed.

\section{Experiments}

The conventional ITO tablets were fabricated by using the sintered-ITO powders. The modified ITO tablets were fabricated by using the nano- and sintered-ITO powders. The sintered-ITO powders were obtained by pressing and sintering the nano-ITO powders at $1200{ }^{\circ} \mathrm{C}$. Then, the nano-and sintered-ITO powders, organic agents and water were grinded, dried and granulated. Finally, the conventional and modified ITO tablets with dimension of $\Phi 25 \times 10 \mathrm{~mm}$ were sintered at $1350^{\circ} \mathrm{C}$ under oxygen atmosphere. The ITO tablets were evaporated by electron beam to analyze the needles and cracks.

The microstructure and ingredients of the needles and tablets were analyzed by scanning electron microscope (SEM) using JSM-5610LV (JEOL) and F50 (FEI Inspect). Thermal conductivity was measured by laser flash thermal constant analyzer (TC-7000, ULVAC-RIKO). Three-point bending strength of the ITO tablets were measured by electronic universal testing machine (Shimadzu, AG-X). The optical 
transmittance in the visible light range was measured by spectrophotometer (V-600, Jasco).

\section{Results and Discussion}

Figure 1 shows the images of surface morphology of the evaporated ITO tablets. As shown in Figure 1a, the protuberances (named needle) erect on the surface of the evaporated conventional ITO tablets. The cracks are also observed and spread out as shown in Figure 1b. Figure 1c shows an integrity appearance of the modified ITO tablets after EB evaporation. The needles and cracks in the conventional ITO tablets would influence the divergence angle of oxide vapor and even generate dust in the coating chamber, which forms particle contamination on the ITO film surface, and deteriorates the electrical and optical properties of GaN-LEDs.

The thermal conductivity of the conventional and modified ITO tablets is 7.2 and $8.5 \mathrm{~W} \cdot \mathrm{m}^{-1} \mathrm{~K}^{-1}$, respectively. The conventional and modified ITO tablets show low thermal conductivity, which indicates that the elimination of cracks is dependent on microstructure rather than the thermal conductivity. Figure 2 shows the SEM images of the conventional ITO tablets. The microstructure indicates that the ITO tablets possesses uniform density at different positions. The size of grains and pores is uniform, and its distribution is homogeneous. This single configuration shows loose structure with weak bonding strength. Thermal shockresistant of this ITO tablets is weak under the bombardment of high energy electron beam during EB evaporation process. Therefore, this homogeneous structure is easily destroyed by thermal shock of EB evaporation or reactive plasma deposition (RPD) process.

Figure 3 shows the SEM images of the modified ITO tablets. As shown in Figure 3a, the microstructure indicates that the modified ITO tablets are composed of dense blocky grains (region A) and loose pores (region B). Figure $3 \mathrm{~b}$ and Figure $3 \mathrm{c}$ indicate that the dense blocky regions resemble the dense structure of an high density ITO ceramic target ${ }^{15}$, a few pores exist in the grain boundaries. Figure $3 d$ and Figure $3 \mathrm{e}$ indicate that the loose porous regions is similar with the microstructure of porous ceramic ${ }^{16}$, a number of pores exist in the grain boundaries. The modified ITO tablets show heterogeneous structure, which provides a way to enhance the bonding strength.

The microstructure models of the conventional and modified ITO tablets are demonstrated in Figure 4. The heterogeneous and porous structure can be considered as an effective solution for resisting thermal shock of the ITO

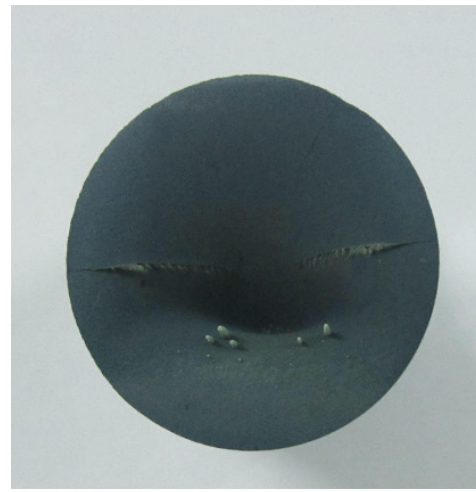

(a)

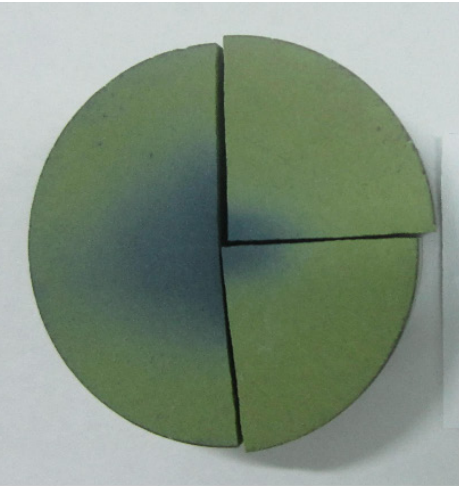

(b)

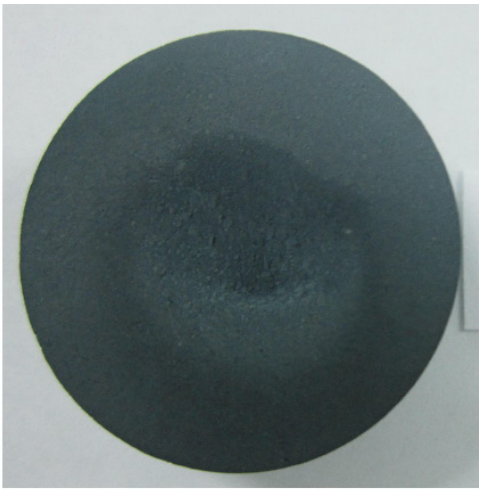

(c)

Figure 1. Images of (a) needles, (b) cracks in conventional ITO tablets, and (c) a complete modified ITO tablet.

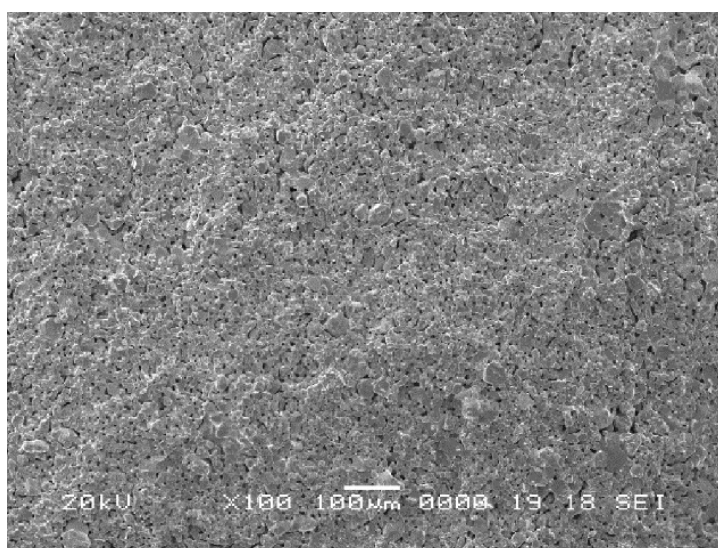

(a)

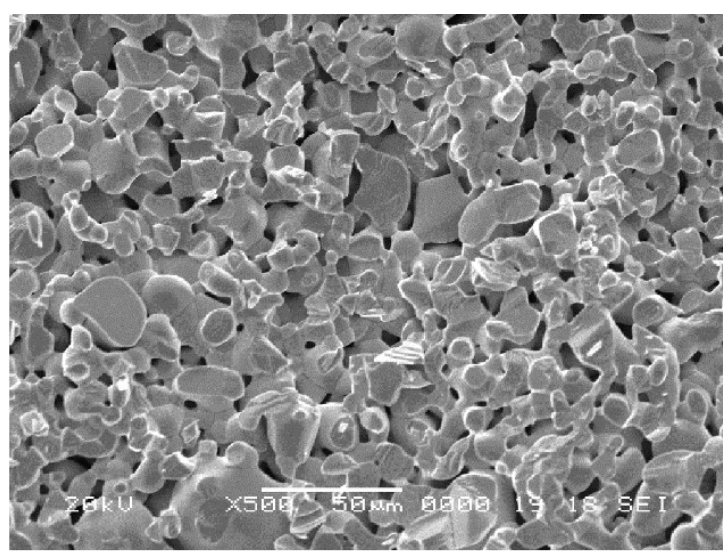

(b)

Figure 2. SEM images of the conventional ITO tablets at (a) low and (b) high magnification. 


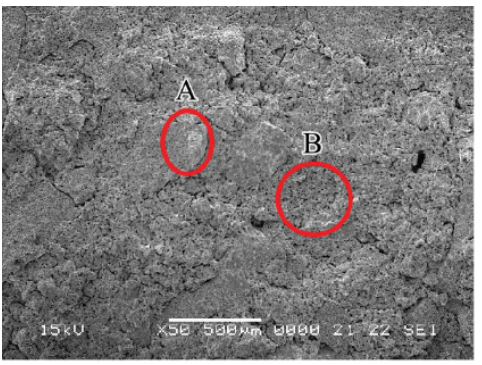

(a)

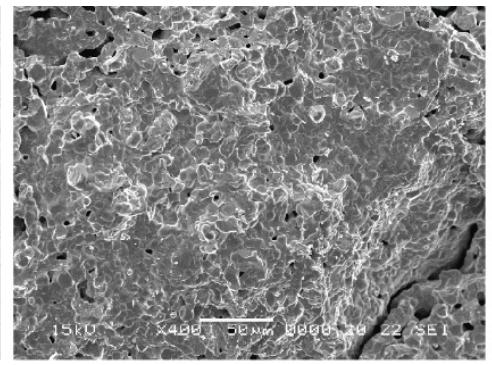

(b)

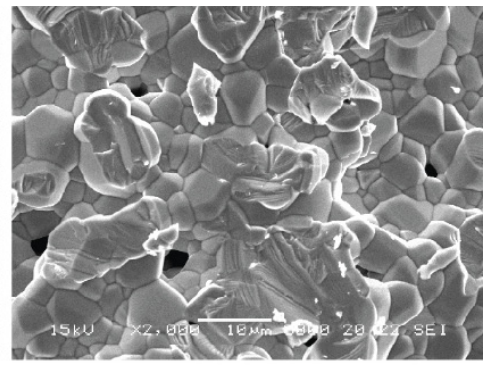

(c)

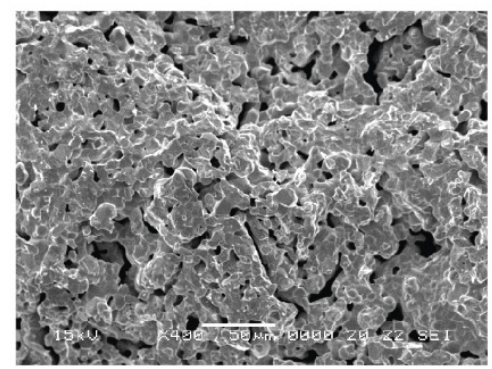

(d)

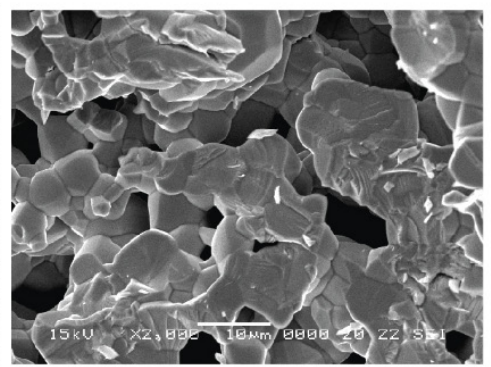

(e)

Figure 3. SEM images of the modified ITO tablets: (a) overall view, (b) dense region (region A), (c) magnification of dense region, (d) loose region (region B), (e) magnification of loose region.

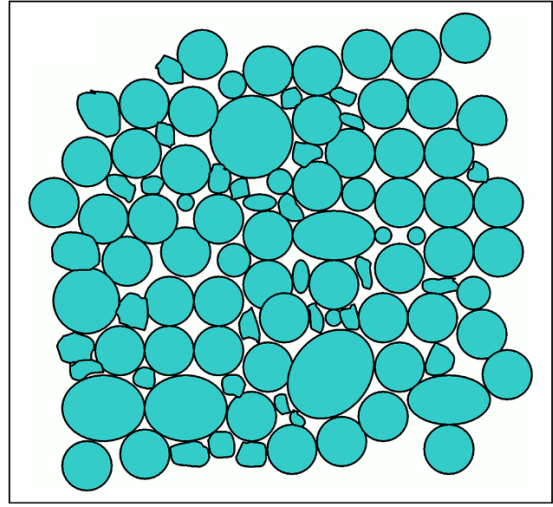

(a)

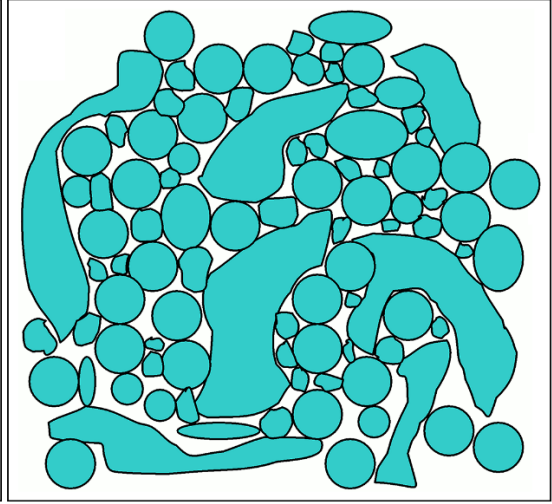

(b)

Figure 4. Microstructure models of (a) conventional and (b) modified ITO tablets.

tablets when they are heated by high energy electron beam. However, the bonding strength of the single structure is weak, which is easily broken under thermal shock. The structure of the modified tablets is reticulated with some skeletons. This three-dimensional reticulated skeleton structure simultaneously solves the problems of thermal expansion and bonding strength. The long strip samples were fabricated to measure three-point bending strength and analyze the bonding strength. The bonding strength of the conventional and modified ITO tablets is about $3.5 \mathrm{MPa}$ and $14.3 \mathrm{MPa}$, which shows an obvious improvement. Consequently, the cracks in the modified ITO tablets can be eliminated during the EB evaporation process.

Figure 5 shows the microstructure of the needles. The middle section of the needle (region B) is composed of large blocky ITO grains, which can be clearly observed in Figure $5 \mathrm{~b}$ and Figure 6 . However, the structure of the bottom section (region A) is consistent with the intrinsic grains of the as-fabricated ITO tablets.

The EDS analysis of the needles, shown in Figure 6, indicates that the blocky needle is composed of indium, tin and silicon. The as-fabricated ITO tablets with a trace of silicon are inevitable, the other impurities are invisible. Therefore, The EDS results indicate that the needles are not caused by impurities. The melted ITO tablet vapor causes abnormal grain growth, which results in blocky needles on the surface.

During EB evaporation process, the EB size is about several millimeters. In order to etch the tablet as large as possible, the EB is usually scanned by applying external scan signals at $x$ and $y$ axes. The signal may be sine, triangle and square waves. The EB scan trace can be described by Lissajous curve. Lissajous curve is the graph of a system of parametric equations ${ }^{17}$ : 


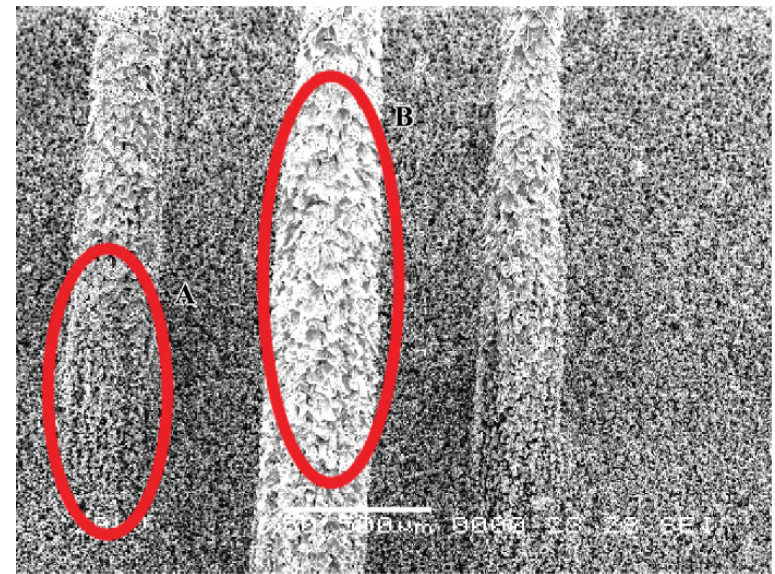

(a)

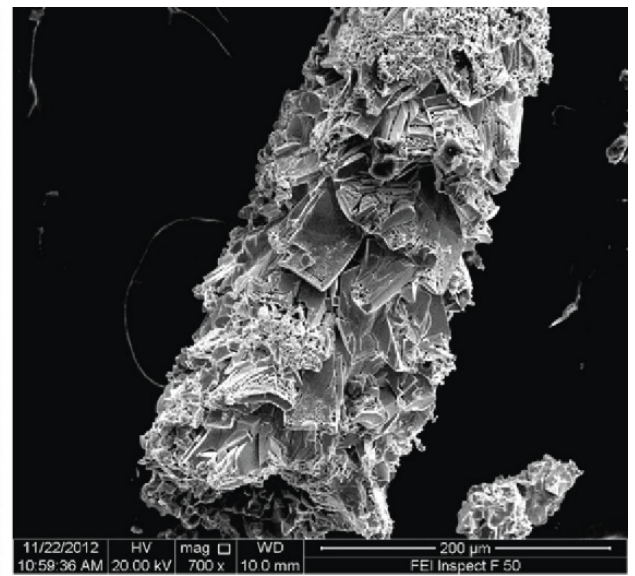

(b)

Figure 5. SEM images of (a) needles and (b) magnification of region B.
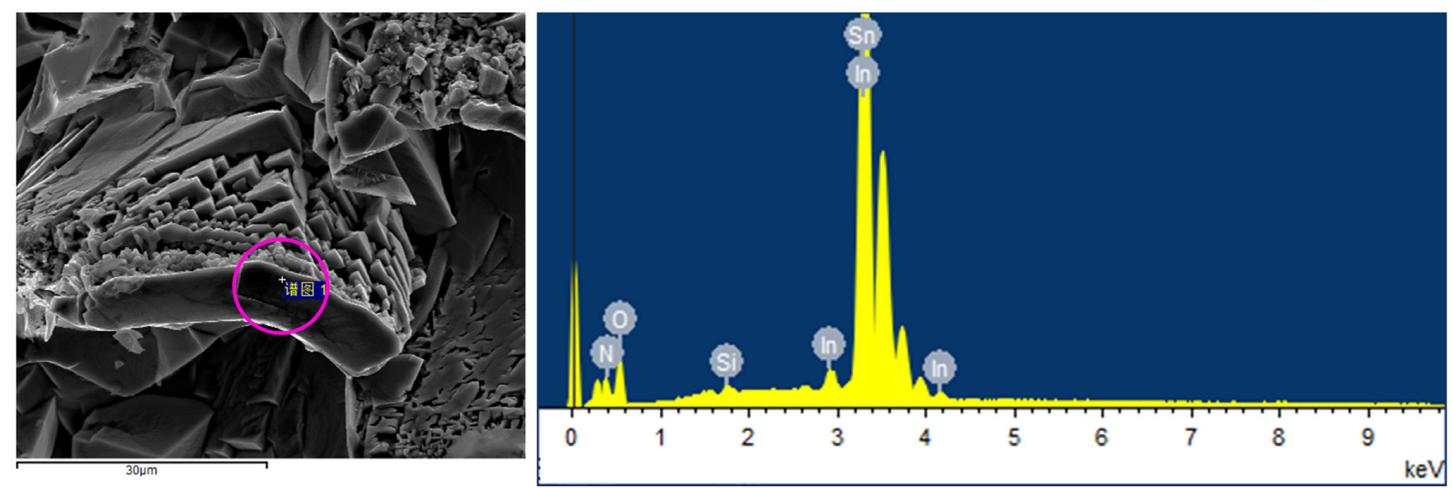

(a)
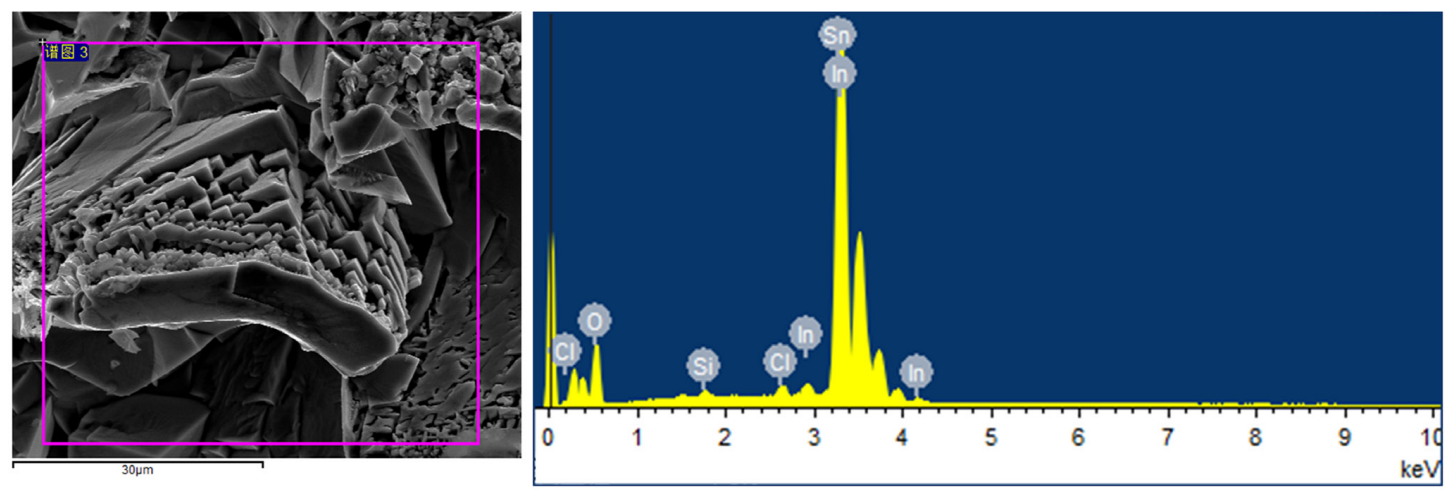

(b)

Figure 6. (a) point measurement and (b) region scan of EDS analysis for needle.

$x(t)=A \sin \left(\omega_{1} t+\varphi_{1}\right)$

$$
y(t)=B \sin \left(\omega_{2} t+\varphi_{2}\right)
$$

Where $\mathrm{A}$ and $\mathrm{B}$ are the amplitude, $\omega_{1}$ and $\omega_{2}$ are the angular frequency, and $\varphi_{1}$ and $\varphi_{2}$ are the phase. The appearance of the Lissajous curve is highly sensitive to the frequency ratio of $\omega_{1} / \omega_{2}$ and the phase difference of $\varphi_{1}-\varphi_{2}$.
Figure 7 a shows nine Lissajous curves, and the more sophisticated curves can be obtained by adjusting the frequency ratio and phase difference. Furthermore, these scan trace curves keep a fixed path and continuously repeat when the scan signals are unchanged. Therefore, the adjustment of the EB size and scan trace is an essential way to resolve the needle problem.

Large size EB and rational frequency ratio can enable the EB to scan the entire surface area of the ITO tablet. However, 


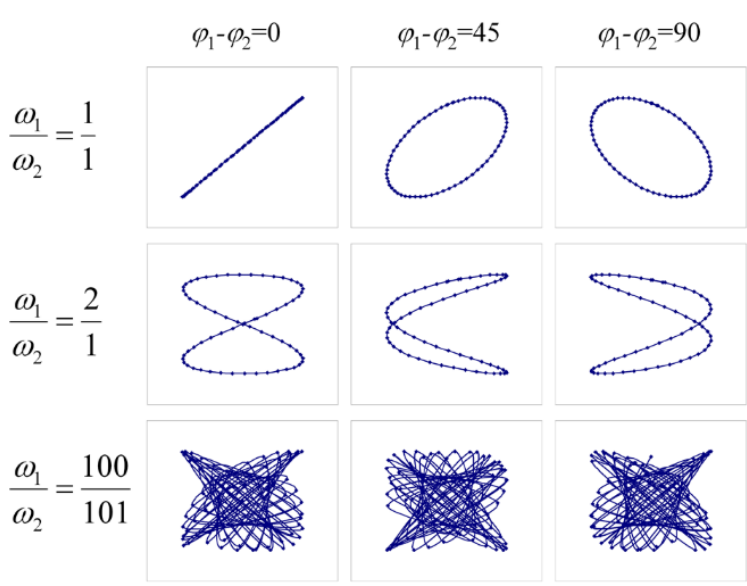

(a)

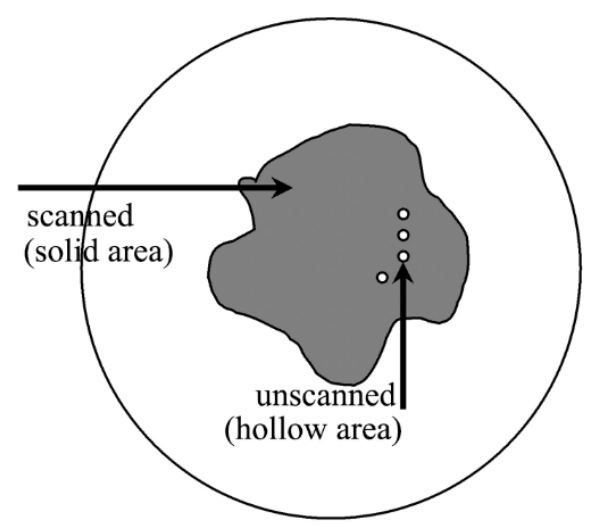

electron beam

(b)

Figure 7. (a) Lissajous curves at different frequency ratios and phase differences, and (b) EB scan model.

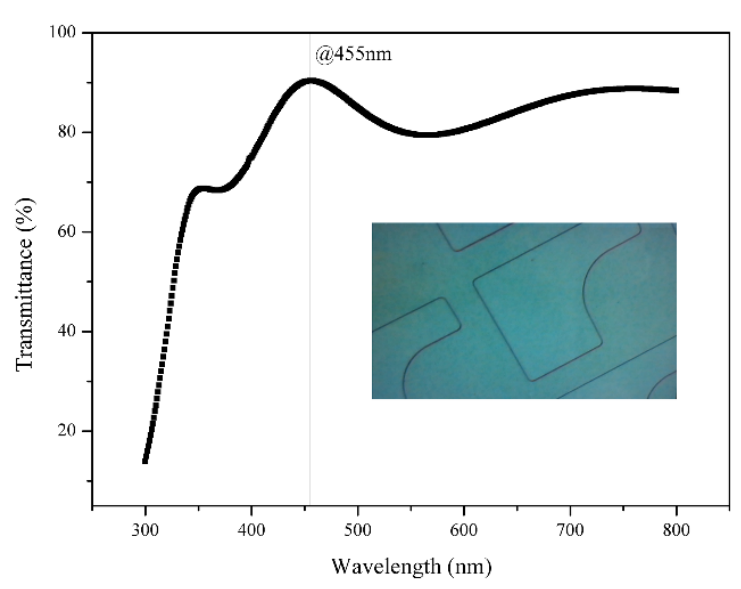

Figure 8. Transmittance of the ITO film and the etched patterns of the ITO electrodes.

the EB size is normally fixed at a value (e.g. $2 \mathrm{~mm}$ ), which can not be adjusted by operator. Therefore, only frequency ratio can be controlled. If the frequency ratio of $\omega_{1} / \omega_{2}$ is inappropriate, some regions of the ITO tablet surface can not be scanned by EB, and these regions can not be gasified by high energy electron beam. Therefore, these regions contribute to the needles as shown in Figure 7b. It is obvious that the quantity and size of needles are closely related with the EB size and frequency ratio. The proposed frequency ratio should have large simple integer ratio $(\mathrm{m} / \mathrm{n}, \mathrm{m}$ and $\mathrm{n}$ is simple integer), such as $30 / 1,44 / 3$.

The ITO film with $250 \mathrm{~nm}$ thickness used for GaN-LED of $455 \mathrm{~nm}$ wavelength was deposited onto glass substrate by

\section{References}

1. Lewis BG and Paine DC. Applications and processing of transparent conducting oxides. MRS Bulletin. 2000; 25(8):2227. http://dx.doi.org/10.1557/mrs2000.147.

2. Xu J, Yang Z, Zhang X, Wang H and Xu H. Structural, electrical, optical properties and reliability of ultra-thin tin doped indium using modified ITO tablet at optimized process parameters. Figure 8 shows the transmittance (including the absorption of glass substrate) of the ITO film and the etched patterns of the ITO electrodes. It can be seen that the ITO film shows ultrahigh transmittance of about $90 \%$ at desired $455 \mathrm{~nm}$, which contributes to increase the output of light at $455 \mathrm{~nm}$. The etched patterns of ITO electrodes indicates that the patterns show sharp edge and have no abnormal spot, crack and etching residue.

\section{Conclusions}

In this work, the needles and cracks of the conventional ITO tablets used for the EB evaporation process were deeply investigated. The formation of the needles during EB evaporation process is mainly due to the EB size and scan trace which controlled by $x$ and $y$ axes scan signals. The needles problem can be solved by controlling the EB scan trace. The conventional tablets composed of homogenous grains and pores show loose microstructure and easily split under thermal shock of the high energy electron beam. The cracks problem can be solved by constructing a three-dimensional reticulated skeleton structure with blocky and porous regions. The $250 \mathrm{~nm}$ thickness ITO film shows high transmittance of $90 \%$ at $455 \mathrm{~nm}$ wavelength of GaN-based LED.

\section{Acknowledgements}

This work is jointly supported by the National Science Foundation of China (21176051, 61361004), Guangxi Key Laboratory of Information Materials Foundation (1210908-204-Z, 131024-Z) and Guangxi Experiment Center of Information Science.

oxide films for touch panels. Journal of Materials Science Materials in Electronics. 2014; 25(4):1792-1797. http://dx.doi. org/10.1007/s10854-014-1800-y.

3. Cesaria M, Caricato AP, Maruccio G and Martino M. Optical analysis of Cr-doped ITO films deposited by double-target laser ablation. Journal of Luminescence. 2015; 162:155-163. http://dx.doi.org/10.1016/j.jlumin.2015.02.047. 
4. Mohamed HA. Effect of substrate temperature on physical properties of $\operatorname{In}_{2} \mathrm{O}_{3}: \mathrm{Sn}$ films deposited by e-beam technique. International Journal of Physical Sciences. 2012; 7(13):21022109.

5. Szkutnik PD, Roussel H, Lahootun V, Mescot X, Weiss F and Jiménez C. Study of the functional properties of ITO grown by metalorganic chemical vapor deposition from different indium and tin precursors. Journal of Alloys and Compounds. 2014; 603:268-273. http://dx.doi.org/10.1016/j.jallcom.2014.03.088.

6. Jafan MM, Zamani-Meymian M, Rahimi R and Rabbani M. Effect of pyrolysis temperature on the electrical, optical, structural, and morphological properties of ITO thin films prepared by a sol-gel spin coating process. Microelectronic Engineering. 2014; 130:40-45. http://dx.doi.org/10.1016/j.mee.2014.09.009.

7. Marikkannu S, Kashif M, Sethupathy N, Vidhya VS, Piraman S, Ayeshamariam A, et al. Effect of substrate temperature on indium tin oxide (ITO) thin films deposited by jet nebulizer spray pyrolysis and solar cell application. Materials Science in Semiconductor Processing. 2014; 27:562-568. http://dx.doi. org/10.1016/j.mssp.2014.07.036.

8. Cho BR and Park IY. Effect of post-annealing on the formation of ITO thin films. Journal of the Korean Physical Society. 2008; 53(1):19-22.

9. Yosvichit B, Horprathum M, Eiamchai P, Patthanasetakul V, Samransuksamer B, Chindaudom P, et al. Effect of emitting current ion source on structural, optical and electrical properties of nanostructured ito thin films by ion-beam assisted evaporation. Advanced Materials Research. 2014; 979:263-266. http://dx.doi. org/10.4028/www.scientific.net/AMR.979.263.

10. Pokaipisit A, Horprathum M and Limsuwan P. Influence of annealing temperature on the properties of ITO films prepared by electron beam evaporation and ion-assisted deposition. Kasetsart Journal. 2008; 42(5):362-366.

11. Kim K-K, Kim H, Lee S-N and Cho S. Structural, optical, and electrical properties of E-beam and sputter-deposited ITO films for LED applications. Electronic Materials Letters. 2011; 7(2):145-149. http://dx.doi.org/10.1007/s13391-011-0610-0.

12. Yamaguchi M, Ide-Ektessabi A, Nomura $\mathrm{H}$ and Yasui $\mathrm{N}$. Characteristics of indium tin oxide thin films prepared using electron beam evaporation. Thin Solid Films. 2004; 447-448:115118. http://dx.doi.org/10.1016/j.tsf.2003.09.033.

13. Chang CS, Chang SJ, Su YK, Kuo CH, Lai WC, Lin YC, et al. High brightness ingan green leds with an ITO on $\mathrm{n}^{++}$-SPS upper contact. IEEE Transactions on Electron Devices. 2003; 50(11):2208-2212. http://dx.doi.org/10.1109/TED.2003.819091.

14. Kuo CH, Chang SJ, Su YK, Chuang RW, Chang CS, Wu LW, et al. Nitride-based near-ultraviolet LEDs with an ITO transparent contact. Materials Science and Engineering B. 2004; 106(1):69-72. http://dx.doi.org/10.1016/j.mseb.2003.09.018.

15. Zhu G, Yang Z, Zhi L, Yang H, Xu H and Yu A. Preparation and Sintering Behavior of the Tin-Doped Indium Oxide Nanopowders. Journal of the American Ceramic Society. 2010; 93(9):25112514. http://dx.doi.org/10.1111/j.1551-2916.2010.03851.x.

16. Sakka Y, Tang F, Fudouzi H and Uchikoshi T. Fabrication of porous ceramics with controlled pore size by colloidal processing. Science and Technology of Advanced Materials. 2005; 6(8):915920. http://dx.doi.org/10.1016/j.stam.2005.07.006.

17. Al-Khazali HAH and Askari MR. Geometrical and graphical representations analysis of lissajous figures in rotor dynamic system. IOSR Journal of Engineering. 2012; 2(05):971-978. http://dx.doi.org/10.9790/3021-0205971978. 